\title{
Design and Adaptation for Cross-Device, Context-dependent User Interfaces
}

Fabio Paternò

CNR-ISTI, HIIS Laboratory

Via Moruzzi 1

56124 Pisa, Italy

fabio.paterno@isti.cnr.it

\begin{abstract}
This tutorial aims to help user interface designers and developers to understand the issues involved in multidevice, context-dependent interactive applications, which can be accessed through wearable, mobile and stationary devices even exploiting different interaction modalities. It will provide a discussion of the possible solutions in terms of concepts, techniques, languages, and tools, with particular attention to Web

environments. The tutorial will deal with the various strategies in order to adapt, distribute, and migrate the user interface according to the context of use.
\end{abstract}

Permission to make digital or hard copies of part or all of this work for personal or classroom use is granted without fee provided that copies are not made or distributed for profit or commercial advantage and that copies bear this notice and the full citation on the first page. Copyrights for third-party components of this work must be honored. For all other uses, contact the Owner/Author.

Copyright is held by the owner/author(s).

CHI'15 Extended Abstracts, Apr 18-23 2015, Seoul, Republic of Korea ACM 978-1-4503-3146-3/15/04

http://dx.doi.org/10.1145/2702613.2706686

\section{Author Keywords}

Multi-device User Interfaces, Distributed and Migratory User Interfaces, Context-dependent Applications

\section{ACM Classification Keywords}

H. 5 Information Interfaces and Presentation; H.5.2 User Interfaces.

\section{Introduction}

Our lives are a multi-device experience where people are surrounded by different types of devices through which they can connect to networks in different ways. However, one big potential source of frustration is that user interfaces are often not able to exploit well the changing interaction resources when users move about and devices available dynamically change, thus generating several issues. In general main issues in multi-device UIs are: poor adaptation to the context of use, lack of coordination among tasks performed through different devices, inadequate support for seamless cross-device task performance. There is a need for better understanding concepts, methods and tools to support designers and developers to obtain user interfaces able to effectively and opportunistically adapt to the various interaction modalities available and support task continuity. 


\section{Content}

After introducing some basic concepts in contextdependent user interfaces and usability criteria that depend on the interaction resources available, I will first explain how the device choice has an influence on the possible tasks to accomplish and how the structure of such tasks can vary in terms of possible secondary tasks, inter-task temporal relations, and content required depending on the device. Next, the discussion will move on to introducing the main motivations and aspects in cross-device user interfaces and the main approaches in authoring them. The tutorial will then provide a brief overview of the results that can be obtained through model-based approaches when multi-device interfaces are considered. Regarding adaptation we will see the various types of user interface adaptation rules that can be applied, and then focus on how to indicate rules to specify context-dependent behaviour. In this respect, some current solutions will be discussed in terms of their usability and expressiveness. The next part of the tutorial will be dedicated to discussing responsive design, how it works, and the aspects that are not supported by this approach. We will see further solutions in terms of multimodal context-dependent adaptation will be discussed. I will then move on to discuss various levels of flexible access in multi-device environments. I will discuss examples for each level of multi-device access, including support from tools and environments publicly available. Regarding distribution of user interface parts across multiple devices, we will discuss various possible solution at design and at run-time. At this point I will review the various possible approaches to migration in multi-device contexts as well. In multi-user contexts there are various privacy aspects that need to be addressed through specific user customizable privacy policies. Security issues will be briefly discussed as well.

\section{Instructor}

Fabio Paternò is Research Director at CNR-ISTI, where he founded and leads the Laboratory on Human Interfaces in Information Systems. in recent years his main research interests have been in concepts and tools for context-dependent applications, model-based design and development, authoring and design of cross-device interactive applications, distributed and migratory interfaces.

\section{References}

[1] The New Multi-screen World: Understanding CrossPlatform Consumer Behavior. Google Research Report, 2012.http://services.google.com/fh/files/misc/multiscre enworld_final.pdf

[2] T.H.Chang, and Y.Li, Deep Shot: A Framework for Migrating Tasks Across Devices Using Mobile Phone Cameras. Proceedings ACM CHI '11, pp. 2163-2172.

[3] G. Ghiani, F. Paternò and C. Santoro, Push and Pull of Web User Interfaces in Multi-Device

Environments, Proceedings AVI 2012, pp.10-17, ACM Press.

[4] L.Frosini, F. Paternò: User Interface Distribution in Multi-Device and Multi-User Environments with Dynamically Migrating Engines. ACM EICS 2014, pp.55-64, ACM Press, Rome, June 2014.

[5] M. Nebeling, T. Mintsi, M. Husmann, M, C. Norrie: Interactive development of cross-device user interfaces. ACM CHI 2014, pp. 2793-2802

6] J. Yang, D. Wigdor: Panelrama: enabling easy specification of cross-device web applications. ACM CHI 2014, Toronto, pp.2783-2792 